\title{
Finite-time attitude synchronization with distributed discontinuous protocols
}

\author{
Jieqiang Wei, Silun Zhang, Antonio Adaldo, Johan Thunberg, Xiaoming Hu, Karl H. Johansson
}

\begin{abstract}
The finite-time attitude synchronization problem is considered in this paper, where the rotation of each rigid body is expressed using the axis-angle representation. Two discontinuous and distributed controllers using the vectorized signum function are proposed, which guarantee almost global and local convergence, respectively. Filippov solutions and non-smooth analysis techniques are adopted to handle the discontinuities. Sufficient conditions are provided to guarantee finite-time convergence and boundedness of the solutions. Simulation examples are provided to verify the performances of the control protocols designed in this paper.
\end{abstract}

Index Terms-Agents and autonomous systems, Finite-time attitude synchronization, Network Analysis and Control, Nonlinear systems

\section{INTRODUCTION}

Originally motivated by aerospace developments in the middle of the last century [5], [15], the rigid body attitude control problem has continued to attract attention with many applications such as aircraft attitude control [2], [32], spacial grabbing technology of manipulators [21], target surveillance by unmanned vehicles [24], and camera calibration in computer vision [20]. Furthermore, the configuration space of rigid-body attitudes is the compact non-Euclidean manifold $S O(3)$, which poses theoretical challenges for attitude control [3].

Here we review some related existing work. As attitude systems evolves on $S O(3)$ - a compact manifold without a boundary-there exists no continuous control law that achieves global asymptotic stability [6]. Hence one has to resort to some hybrid or discontinuous approaches. In [17], exponential stability is guaranteed for the tracking problem for a single attitude. The coordination of multiple attitudes is of high interest both in academic and industrial research, e.g., [11], [26], [29]. In [18] the authors considered the synchronization problem of attitudes under a leader-follower architecture. In [23], the authors provided a local result on attitude synchronization. Based on a passivity approach, 25 proposed a consensus control protocol for multiple rigid bodies with attitudes represented by modified Rodrigues parameters. In [31], the authors provided a control protocol in discrete time that achieves almost global synchronization, but it requires global knowledge of the graph topology. Although there exists

*This work is supported by Knut and Alice Wallenberg Foundation, Swedish Research Council, and Swedish Foundation for Strategic Research.

J. Wei, A. Adaldo and K.H. Johansson are with the ACCESS Linnaeus Centre, School of Electrical Engineering. S. Zhang and X. Hu are with School of engineering sciences. KTH Royal Institute of Technology, SE-100 44 Stockholm, Sweden. \{jieqiang, silunz, adaldo, hu, kallej\}akth. se. Johan Thunberg is with Luxembourg Centre for Systems Biomedicine, Universit du Luxembourg,6, avenue du Swing, L4367 Belvaux. \{johan.thunberg\}@uni.lu no continuous control law that achieves global asymptotic stability, a methodology based on the axis-angle representation obtains almost global stability for attitude synchronization under directed and switching interconnection topologies is proposed in [30]. These control laws were later generalized to include various types of vector representations including the Rodrigues Parameters and Unit Quaternions [29]. Besides these agreement results, [16], [28], [34] provided distributed schemes for more general formation control of attitude in space $S O(3)$.

Among all the properties of attitude synchronization schemes, the finite-time convergence is an important one, because in practice it is desired that the system reaches the target configuration within a certain time-interval; consider, for instance, satellites in space that shall face a certain direction as they move in their orbits, or cameras that shall reach a certain formation to quickly follow an object. So far, finitetime attitude control problems are studied in different settings, e.g., [12], [35]. In [12], finite-time attitude synchronization was investigated in a leader-follower architecture, namely all the followers tracking the attitude of the leader. In [35], quaternion representation was employed for finite-time attitude synchronization. Both works used continuous control protocols with high-gain.

In this paper, we shall focus on the finite-time attitude synchronization problem, based on the axis-angle representations of the rotations without a leader-follower architecture, using discontinuous control laws. Two intuitive control schemes are proposed. The first scheme employs a direction-preserving sign function to guarantee finite-time synchronization almost globally, namely, the convergence holds for almost all the initial conditions. The other scheme, motivated by binary controllers for scalar multi-agent systems, e.g., [7], [19], [9], [14], uses the component-wise sign function. Compared to the first scheme, the second one is more coarse, in the sense that only finite number of control outputs are employed, and guarantees finite-time convergence locally. Since these control schemes are discontinuous, nonsmooth analysis is employed throughout the paper.

The structure of the paper is as follows. In Section II we review some results for the axis-angle representation for rotations in $S O(3)$ and introduce some terminologies and notations in the context of graph theory and discontinuous dynamical systems. Section III presents the problem formulation of the finite-time attitude synchronization problem. The main results of the stability analysis of the finite-time convergence are presented in Section IV] where an almost global and local stability are given in Subsection IV-A and IV-B respectively. Then, in Section $\mathrm{V}$, the paper is concluded.

Notation. With $\mathbb{R}_{-}, \mathbb{R}_{+}, \mathbb{R}_{\geqslant 0}$ and $\mathbb{R}_{\leqslant 0}$ we denote the sets 
of negative, positive, non-negative, non-positive real numbers, respectively. The rotation group $S O(3)=\left\{R \in \mathbb{R}^{3 \times 3}\right.$ : $\left.R R^{\top}=I, \operatorname{det} R=1\right\}$. The vector space of real $n$ by $n$ skew symmetric matrices is denoted as $\mathfrak{s o}(3)$. $\|\cdot\|_{p}$ denotes the $\ell_{p}$-norm and the $\ell_{2}$-norm is denoted simply as $\|\cdot\|$ without a subscript.

\section{PRELIMINARIES}

In this section, we briefly review some essentials about rigid body attitudes [27], graph theory [4], and give some definitions for Filippov solutions [13].

Next lemma follows from Euler's Rotation Theorem.

Lemma 1. The exponential map

$$
\exp : \mathfrak{s o}(3) \rightarrow S O(3)
$$

is surjective.

For any $p=\left[p_{1}, p_{2}, p_{3}\right]^{\top} \in \mathbb{R}^{3}$ and $\hat{p} \in \mathfrak{s o}(3)$ given as

$$
\hat{p}:=\left(\begin{array}{ccc}
0 & -p_{3} & p_{2} \\
p_{3} & 0 & -p_{1} \\
-p_{2} & p_{1} & 0
\end{array}\right),
$$

Rodrigues' formula is the right-hand side of

$$
\exp (\hat{p})=I_{3}+\frac{\sin (\theta)}{\theta} \hat{p}+\frac{1-\cos (\theta)}{\theta^{2}}(\hat{p})^{2},
$$

where $\theta=\|p\|$ and $\exp (\hat{p})$ is the rotation matrix through an angle $\theta$ anticlockwise about the axis $p$. For $R \in S O(3)$ where $R$ is not symmetric, we define the inverse of exp as

$$
\log (R)=\frac{\theta}{2 \sin (\theta)}\left(R-R^{\top}\right)
$$

where $\theta=\arccos \left(\frac{\operatorname{trace}(R)-1}{2}\right)$. We define $\log \left(I_{3}\right)$ as the zero matrix in $\mathbb{R}^{3 \times 3}$. Note that (4) is not defined for $\theta=\pi$. The Riemannian metric for $S O(3)$ is defined as $d_{R}\left(R_{1}, R_{2}\right)=$ $\frac{1}{\sqrt{2}}\left\|\log \left(R_{1}^{-1} R_{2}\right)\right\|_{F}$ where $\|\cdot\|_{F}$ is the Frobenius norm.

One important relation between $S O(3)$ and $\mathbb{R}^{3}$ is that the open ball $B_{\pi}(I)$ in $S O(3)$ with radius $\pi$ around the identity, which is almost the whole $S O(3)$, is diffeomorphic to the open ball $B_{\pi}(0)$ in $\mathbb{R}^{3}$ via the logarithmic and the exponential map defined in (4) and (3).

An undirected graph $\mathcal{G}=(\mathcal{I}, \mathcal{E})$ consists of a finite set of nodes $\mathcal{I}=\{1,2, \ldots, n\}$ and a set of edges $\mathcal{E} \in \mathcal{I} \times \mathcal{I}$ of unordered pairs of $\mathcal{I}$. To each edge $(i, j) \in \mathcal{E}$, we associate a weight $w_{i j}>0$. The weighted adjacency matrix $A=\left[a_{i j}\right] \in$ $\mathbb{R}^{n \times n}$ is defined by $a_{i j}=a_{j i}=w_{i j}$ if $(i, j) \in \mathcal{E}$ and $a_{j i}=0$ otherwise. Note that $A=A^{\top}$ and that $a_{i i}=0$ as no self-loops are allowed. For each node $i \in \mathcal{I}$, its degree $d_{i}$ is defined as $d_{i}=\sum_{j=1}^{n} a_{i j}$. The graph Laplacian $L$ is defined as $L=$ $\Delta-A$ where $\Delta$ is a diagonal matrix such that $\Delta_{i i}=d_{i}$. As a result, $L \mathbb{1}=\mathbf{0}$. We denote the set of neighbors of node $i$ as $N_{i}=\left\{j \in \mathcal{I} \mid w_{i j}>0\right\}$. If the edges are ordered pairs of $\mathcal{I}$, the graph $\mathcal{G}$ is called a directed graph, or digraph for short. An edge of a digraph $\mathcal{G}$ is denoted by $(i, j)$ (with $i \neq j$ ) representing the tail vertex $i$ and the head vertex $j$ of this edge. A digraph with unit weights is completely specified by its incidence matrix $B \in \mathbb{R}^{n \times m}$, where $|\mathcal{E}|=m$, with $B_{i j}$ equal to -1 if the $j$ th edge is towards vertex $i$, and equal to 1 if the $j$ th edge is originating from vertex $i$, and 0 otherwise. The incidence matrix for undirected graphs is defined by adding arbitrary orientations to the edges of the graph. Finally, we say that a graph $\mathcal{G}$ is connected if, for any two nodes $i$ and $j$, there exists a sequence of edges that connects them. In order to simplify the notation in the proofs, we set the weight $w_{i j}$ to be one. All the results in this paper hold for the general case where the $w_{i j}$ 's are elements in $R_{+}$.

In the remainder of this section, we discuss Filippov solutions. Let $f$ be a map from $\mathbb{R}^{m}$ to $\mathbb{R}^{n}$ and let $2^{\mathbb{R}^{n}}$ denote the collection of all subsets of $\mathbb{R}^{n}$. The Filippov set-valued map of $f$, denoted $\mathcal{F}[f]: \mathbb{R}^{m} \rightarrow 2^{\mathbb{R}^{n}}$, is defined as

$$
\mathcal{F}[f](x):=\bigcap_{\delta>0} \bigcap_{\mu(S)=0} \overline{\operatorname{co}}\{f(B(x, \delta) \backslash S)\},
$$

where $S$ is a subset of $\mathbb{R}^{m}, \mu$ denotes the Lebesgue measure, $B(x, \delta)$ is the ball centered at $x$ with radius $\delta$ and $\overline{\mathrm{co}}\{\mathcal{X}\}$ denotes the convex closure of a set $\mathcal{X}$. If $f$ is continuous at $x$, then $\mathcal{F}[f](x)$ contains only the point $f(x)$.

A Filippov solution of the differential equation $\dot{x}(t)=$ $f(x(t))$ on $[0, T] \subset \mathbb{R}$ is an absolutely continuous function $x:[0, T] \rightarrow \mathbb{R}^{n}$ that satisfies the differential inclusion

$$
\dot{x}(t) \in \mathcal{F}[f](x(t))
$$

for almost all $t \in[0, T]$. A Filippov solution is maximal if it cannot be extended forward in time, that is, if it is not the result of the truncation of another solution with a larger interval of definition. Next, we introduce invariant sets, which will play a key part further on. Since Filippov solutions are not necessarily unique, we need to specify two types of invariant sets. A set $\mathcal{R} \subset \mathbb{R}^{n}$ is called weakly invariant if, for each $x_{0} \in \mathcal{R}$, at least one maximal solution of (5) with initial condition $x_{0}$ is contained in $\mathcal{R}$. Similarly, $\mathcal{R} \subset \mathbb{R}^{n}$ is called strongly invariant if, for each $x_{0} \in \mathcal{R}$, every maximal solution of (5) with initial condition $x_{0}$ is contained in $\mathcal{R}$. For more details, see [10], [13]. We use the same definition of regular function as in [8] and recall that any convex function is regular.

For $V: \mathbb{R}^{n} \rightarrow \mathbb{R}$ locally Lipschitz, the generalized gradient $\partial V: \mathbb{R}^{n} \rightarrow 2^{\mathbb{R}^{n}}$ is defined by

$$
\partial V(x):=\operatorname{co}\left\{\lim _{i \rightarrow \infty} \nabla V\left(x_{i}\right) \mid x_{i} \rightarrow x, x_{i} \notin S \cup \Omega_{f}\right\},
$$

where $\nabla$ is the gradient operator, $\Omega_{f} \subset \mathbb{R}^{n}$ is the set of points where $V$ fails to be differentiable and $S \subset \mathbb{R}^{n}$ is a set of measure zero that can be arbitrarily chosen to simplify the computation, since the resulting set $\partial V(x)$ is independent of the choice of $S$ [8].

Given a set-valued map $\mathcal{T}: \mathbb{R}^{n} \rightarrow 2^{\mathbb{R}^{n}}$, the set-valued Lie derivative $\mathcal{L}_{\mathcal{T}} V: \mathbb{R}^{n} \rightarrow 2^{\mathbb{R}^{n}}$ of a locally Lipschitz function $V: \mathbb{R}^{n} \rightarrow \mathbb{R}$ with respect to $\mathcal{T}$ at $x$ is defined as

$$
\begin{gathered}
\mathcal{L}_{\mathcal{T}} V(x):=\{a \in \mathbb{R} \mid \exists \nu \in \mathcal{T}(x) \text { such that } \\
\left.\zeta^{T} \nu=a, \forall \zeta \in \partial V(x)\right\} .
\end{gathered}
$$

If $\mathcal{T}(x)$ is convex and compact $\forall x \in \mathbb{R}^{n}$, then $\mathcal{L}_{\mathcal{T}} V(x)$ is a compact interval in $\mathbb{R}$, possibly empty.

The $i$ th row of a matrix $M$ is denoted as $M_{i}$. For any matrix $M$, we denote $M \otimes I_{3}$ as $\hat{M}$ and $M_{i} \otimes I$ as $\hat{M}_{i}$. A 
positive definite and semidefinite (symmetric) matrix $M$ is denoted as $M>0$ and $M \geqslant 0$, respectively. The vectors $e_{1}, e_{2}, \ldots, e_{n}$ denote the canonical basis of $\mathbb{R}^{n}$. The vectors $\mathbb{1}_{n}$ and $\mathbf{0}_{n}$ represents a $n$-dimensional column vector with each entry being 1 and 0 , respectively. In this paper, we define the direction-preserving signum as

$$
\operatorname{sign}(w)= \begin{cases}\frac{w}{\|w\|} & \text { if } w \neq \mathbf{0} \\ 0 & \text { if } w=\mathbf{0}\end{cases}
$$

for $w \in \mathbb{R}^{k}$. The component-wise signum is denoted as

$$
\operatorname{sign}_{c}(w)=\left[\operatorname{sign}\left(w_{1}\right), \ldots, \operatorname{sign}\left(w_{k}\right)\right]^{\top},
$$

where $w=\left[w_{1}, w_{2}, \ldots, w_{k}\right]^{\top}$. Notice that for scalars, these two signum functions coincide. Furthermore, component-wise signum is coarser than direction-preserving in the sense that there is only a finite number of elements in the range for a fixed dimension $k$.

\section{PROBLEM FORMULATION}

We consider a system of $n$ agents (rigid bodies). We denote the world frame as $\mathcal{F}_{w}$ and the instantaneous body frame of agent $i$ as $\mathcal{F}_{i}$ where $i \in \mathcal{I}=\{1, \ldots, n\}$. Let $R_{i}(t) \in S O(3)$ be the attitude of $\mathcal{F}_{i}$ relative to $\mathcal{F}_{w}$ at time $t$, and, when $R_{i}(t) \in B_{\pi}(I)$, the corresponding axis-angle representation $x_{i}(t) \in \mathbb{R}^{3}$ be given by

$$
\hat{x}_{i}(t)=\log \left(R_{i}(t)\right) .
$$

The kinematics of $x_{i}$ is given by [27]

$$
\dot{x}_{i}=L_{x_{i}} \omega_{i}, \quad i \in \mathcal{I}
$$

where $\omega_{i}$ is the control signal corresponding to the instantaneous angular velocity of $\mathcal{F}_{i}$ relative to $\mathcal{F}_{w}$ expressed in the frame $\mathcal{F}_{i}$, and the transition matrix $L_{x_{i}}$ is defined as

$$
\begin{aligned}
L_{x_{i}} & =I_{3}+\frac{\hat{x}_{i}}{2}+\left(1-\frac{\operatorname{sinc}\left(\left\|x_{i}\right\|\right)}{\operatorname{sinc}^{2}\left(\frac{\left\|x_{i}\right\|}{2}\right)}\right)\left(\frac{\hat{x}_{i}}{\left\|x_{i}\right\|}\right)^{2} \\
& =\frac{\operatorname{sinc}\left(\left\|x_{i}\right\|\right)}{\operatorname{sinc}^{2}\left(\frac{\left\|x_{i}\right\|}{2}\right)} I_{3}+\left(1-\frac{\operatorname{sinc}\left(\left\|x_{i}\right\|\right)}{\operatorname{sinc}^{2}\left(\frac{\left\|x_{i}\right\|}{2}\right)}\right) \frac{x_{i} x_{i}^{\top}}{\left\|x_{i}\right\|^{2}}+\frac{\hat{x}_{i}}{2} \\
& =: L_{x_{i}}^{1}+\frac{\hat{x}_{i}}{2}
\end{aligned}
$$

where $\operatorname{sinc}(\alpha)$ is defined as $\alpha \operatorname{sinc}(\alpha)=\sin (\alpha)$ for all $\alpha \neq 0$ and $\operatorname{sinc}(0)=1$, see [27]. Note that for $\left\|x_{i}\right\| \in[0, \pi]$, the function $\frac{\operatorname{sinc}\left(\left\|x_{i}\right\|\right)}{\operatorname{sinc}^{2}\left(\frac{\left\|x_{i}\right\|}{2}\right)}$ is concave and belongs to $[0,1]$. Then the symmetric part of $L_{x_{i}}$, denoted by $L_{x_{i}}^{1}$, is positive semidefinite. More precisely, $L_{x_{i}}^{1}>0$ if $\left\|x_{i}\right\| \in[0,2 \pi)$. Moreover, $L_{x_{i}}$ is Lipschitz on $B_{r}(0)$ for any $0<r<\pi$ (see [30]).

The system (11) can be written in a compact form as

$$
\dot{x}=L_{x} \omega
$$

where

$$
\begin{aligned}
x & =\left[x_{1}^{\top}, \ldots, x_{n}^{\top}\right]^{\top}, \\
L_{x} & =\operatorname{blockdiag}\left(L_{x_{1}}, \ldots, L_{x_{n}}\right), \\
\omega & =\left[\omega_{1}^{\top}, \ldots, \omega_{n}^{\top}\right]^{\top} .
\end{aligned}
$$

For the multi-agent system (13), we assume that the agents can communicate with each other about the state variables $x_{i}$ via an undirected connected graph $\mathcal{G}$. The aim is to design control protocols for $\omega$ such that the absolute rotations of all agents converge to a common rotation in the world frame $\mathcal{F}_{w}$ in finite time, i.e.,

$$
\exists T>0, \bar{R} \in S O(3) \text { s.t. } R_{i} \rightarrow \bar{R}, \forall i \in \mathcal{I} \text {, as } t \rightarrow T \text {. }
$$

This is equivalent to that $x$ converges to the consensus space

$$
\mathcal{C}=\left\{x \in \mathbb{R}^{3 n} \mid \exists \bar{x} \in \mathbb{R}^{3} \text { such that } x=\mathbb{1}_{n} \otimes \bar{x}\right\}
$$

in finite time. We shall propose two distributed controllers that achieve this goal.

\section{MAIN RESULT}

In this section, we shall first present a control law that guarantees that the rotations of all the rigid bodies converge to a common rotation for any initial condition $R_{i}(0) \in B_{\pi}(I) \subset$ $S O(3)$ for all $i \in \mathcal{I}$. Note that this initial condition in $S O(3)$ is equivalent to $\left\|x_{i}(0)\right\|<\pi$ under the axis-angle representation. In order to avoid the singularity of the logarithmic map (4), the control law makes sure that the constraint $\left\|x_{i}(t)\right\|<\pi$ is met for all $i \in \mathcal{I}$ and for all time $t>0$. We consider controllers of the following form

$$
\omega_{i}=f_{i}\left(\sum_{j \in N_{i}}\left(x_{j}-x_{i}\right)\right), \quad i \in \mathcal{I},
$$

with maps $f_{i}: \mathbb{R}^{3} \rightarrow \mathbb{R}^{3}$ and the elements in the set $N_{i}$ are the neighbors of agent $i$. Now the closed-loop system can be written in a compact form as

$$
\dot{x}=L_{x} f(-\hat{L} x)
$$

where $f(y)=\left[f_{1}^{\top}\left(y_{1}\right), \ldots, f_{n}^{\top}\left(y_{n}\right)\right]^{\top}, \hat{L}=L \otimes I_{3}$ and $L$ is the Laplacian of the graph. Our control design is based on the signum function. More precisely, we consider the case when some of the functions $f_{i}$ are $\operatorname{sign}$ or $\operatorname{sign}_{c}$, while the others satisfy certain continuity assumptions to be defined in the following subsections. We propose two control protocols which guarantee almost global, in the sense of $R_{i}(0) \in B_{\pi}(I) \subset S O(3)$, and local convergence, respectively. As discontinuities are introduced into (18) by the signum functions, we shall understand the trajectories in the sense of Filippov, namely an absolutely continuous function $x(t)$ satisfying the differential inclusion

$$
\begin{aligned}
\dot{x} & \in \mathcal{F}\left[L_{x} f(-\hat{L} x)\right](x) \\
& =L_{x} \mathcal{F}[f(-\hat{L} x)](x) \\
& =: \mathcal{F}_{1}(x)
\end{aligned}
$$

for almost all time, where we used Theorem 1(5) in [22].

\section{A. Control law for global convergence}

In this subsection, we shall design a controller such that finite-time synchronization is achieved for any initial condition $R_{i}(0) \in B_{\pi}(I) \subset S O(3)$ by using the direction preserving sign defined in (8). It might seem natural to let $f_{i}=$ sign for all $i \in \mathcal{I}$. However, the following example shows that this 
simple controller does not guarantee $\left\|x_{i}(t)\right\|<\pi, \forall t>0$ for all Filippov solutions.

Example 1. Consider the system

$$
\begin{aligned}
& \dot{x}_{1}=L_{x_{1}} \operatorname{sign}\left(x_{2}+x_{3}-2 x_{1}\right) \\
& \dot{x}_{2}=L_{x_{2}} \operatorname{sign}\left(x_{1}+x_{3}-2 x_{2}\right) \\
& \dot{x}_{3}=L_{x_{3}} \operatorname{sign}\left(x_{2}+x_{1}-2 x_{3}\right)
\end{aligned}
$$

defined on a complete graph. We show that for $t_{0}$ such that $x\left(t_{0}\right) \in \mathcal{C}$, the trajectories can violate the constraints $\left\|x_{i}(t)\right\|<\pi, i \in \mathcal{I}$, for some $t>t_{0}$.

First, by Theorem 1(1) in [22], we have for any $x \in \mathcal{C}$, there exists an $\varepsilon$, independent of $x$, such that the ball $B_{\varepsilon}(0) \subset$ $\mathcal{F}[\operatorname{sign}(-\hat{L} x)](x) \subset \mathbb{R}^{9}$. Second, suppose $x\left(t_{0}\right)=\mathbb{1} \otimes \bar{x}$ for some $\bar{x} \in \mathbb{R}^{3}$. Then there exists $0<\varepsilon_{1}<\varepsilon$ such that the vector $\varepsilon_{1} \mathbb{1} \otimes \frac{\bar{x}}{\|\bar{x}\|} \in B_{\varepsilon}(0)$. Hence,

$$
x(t)=\mathbb{1} \otimes\left(\left(t-t_{0}\right) \varepsilon_{1} \frac{\bar{x}}{\|\bar{x}\|}+\bar{x}\right), t \geqslant t_{0}
$$

is a Filippov solution. Indeed,

$$
\begin{aligned}
\dot{x}(t) & =\varepsilon_{1} \mathbb{1} \otimes \frac{\bar{x}}{\|\bar{x}\|} \\
& =L_{x(t)} \varepsilon_{1} \mathbb{1} \otimes \frac{\bar{x}}{\|\bar{x}\|} \\
& \in L_{x(t)} \mathcal{F}[\operatorname{sign}(-\hat{L} x)](x(t))
\end{aligned}
$$

where the second equality follows from

$$
\begin{aligned}
L_{x(t)} \varepsilon_{1} \mathbb{1} \otimes \frac{\bar{x}}{\|\bar{x}\|} & =L_{x(t)} x(t) \frac{\varepsilon_{1}}{\|\bar{x}\|+\left(t-t_{0}\right) \varepsilon_{1}} \\
& =x(t) \frac{\varepsilon_{1}}{\|\bar{x}\|+\left(t-t_{0}\right) \varepsilon_{1}} \\
& =\varepsilon_{1} \mathbb{1} \otimes \frac{\bar{x}}{\|\bar{x}\|} .
\end{aligned}
$$

Here we used the fact that $L_{x(t)} x(t)=x(t)$. Then for large enough $t,\left\|x_{i}(t)\right\|$ can be larger than $\pi$. The solutions of the type $\mathbb{1} \otimes \eta(t)$ with $\eta(t)$ a non-constant function is called sliding consensus.

The previous example motivates us to consider the following assumption.

Assumption 1. For some set $\mathcal{I}_{c} \subset \mathcal{I}$, the function $f$ in satisfies the following conditions:

(i) For $i \in \mathcal{I}_{c}, f_{i}: \mathbb{R}^{k} \rightarrow \mathbb{R}^{k}$ is locally Lipschitz continuous and satisfies $f_{i}(\mathbf{0})=\mathbf{0}$ and $f_{i}(y)^{\top} y=\left\|f_{i}(y)\right\|\|y\| \neq 0$ for all $y \neq \mathbf{0}$;

(ii) For $i \in \mathcal{I} \backslash \mathcal{I}_{c}$, the function $f_{i}=$ sign.

Note that Condition ( $i$ ) in Assumption 1 corresponds to that $f_{i}$ is direction preserving.

Before showing the result for finite-time convergence, we formulate a condition for the controller (17) satisfying Assumption 1 such that the set $\left\{x \mid\left\|x_{i}\right\|<\pi\right\}$ is strongly invariant for the dynamics (18).

Lemma 2. Consider the differential inclusion (19) satisfying Assumption 1. If one of the following two conditions is satisfied

(i) $|\mathcal{I}|=2$ and $\left|\mathcal{I}_{c}\right|=0$;

(ii) $|\mathcal{I}| \geqslant 2$ and $\left|\mathcal{I}_{c}\right| \geqslant 1$, then the set $\mathcal{S}_{1}(C):=\left\{x \in \mathbb{R}^{3 n} \mid\left\|x_{i}\right\| \leqslant C, i \in \mathcal{I}\right\}$, where $C<\pi$ is a constant, is strongly invariant. This implies that $B_{\pi}(I)^{n}$ is strongly invariant.

Proof. We use a Lyapunov-like argument to prove that for any initial condition in $\mathcal{S}_{1}(C)$, all the solutions of (19) will remain within the set.

Consider the Lyapunov function candidate $V(x)=$ $\max _{i \in \mathcal{I}}\left\|x_{i}\right\|^{2}$. Notice that $V$ is convex, hence regular. Let

$$
\alpha(x)=\left\{i \in \mathcal{I} \mid\left\|x_{i}\right\|_{2}^{2}=V(x)\right\} .
$$

The generalized gradient of $V$ is given as

$$
\partial V(x)=\operatorname{co}\left\{e_{i} \otimes x_{i} \mid i \in \alpha(x)\right\} .
$$

Next, let $\Psi$ be defined as

$$
\Psi=\left\{t \geqslant 0 \mid \text { both } \dot{x}(t) \text { and } \frac{d}{d t} V(x(t)) \text { exist }\right\} .
$$

Since $x$ is absolutely continuous (by definition of Filippov solutions) and $V$ is locally Lipschitz, by Lemma 1 in [1] it follows that $\Psi=\mathbb{R}_{\geqslant 0} \backslash \bar{\Psi}$ for a set $\bar{\Psi}$ of measure zero and that

$$
\frac{d}{d t} V(x(t)) \in \mathcal{L}_{\mathcal{F}_{1}} V(x(t))
$$

for all $t \in \Psi$, so that the set $\mathcal{L}_{\mathcal{F}_{1}} V(x(t))$ is nonempty. For $t \in \bar{\Psi}$, we have that $\mathcal{L}_{\mathcal{F}_{1}} V(x(t))$ is empty, and hence $\max \mathcal{L}_{\mathcal{F}_{1}} V(x(t))=-\infty<0$ by definition. Therefore, we only consider $t \in \Psi$ in the rest of the proof.

By using Theorem 1(4) and (5) in [22], the differential inclusion can be enlarged as follows

$$
\begin{aligned}
\dot{x} & \in \mathcal{F}_{1}(x) \\
& \subset \underset{i=1}{\times} \mathcal{F}\left[L_{x_{i}} f_{i}\left(-\hat{L}_{i} x\right)\right](x) \\
& =\underset{i=1}{\times} L_{x_{i}} \mathcal{F}\left[f_{i}\left(-\hat{L}_{i} x\right)\right](x) \\
& =\underset{i=1}{\times} L_{x_{i}} \mathcal{F}\left[f_{i}\right]\left(-\hat{L}_{i} x\right) \\
& =: \mathcal{F}_{2}(x),
\end{aligned}
$$

where the first equality follows from Assumption 1 and the fact that $L_{x_{i}}$ is continuous for $x_{i}$ with $\left\|x_{i}\right\|<2 \pi$, thus we can use Theorem 1(5) in [22]. Moreover, we obtain that $\mathcal{L}_{\mathcal{F}_{1}} V(x(t)) \subset$ $\mathcal{L}_{\mathcal{F}_{2}} V(x(t))$ for all $t \geqslant 0$. For the rest of the proof, we shall show $\mathcal{L}_{\mathcal{F}_{2}} V(x(t)) \subset \mathbb{R}_{\leqslant 0}$ by considering two cases.

Case 1: For $x \in \mathcal{C}$, i.e., $\alpha(x)=\mathcal{I}$, the following two subcases can be distinguished.

(i) $|\mathcal{I}| \geqslant 2$ and $\left|\mathcal{I}_{c}\right| \geqslant 1$. There is $i \in \mathcal{I}$ such that $f_{i}$ is locally Lipschitz and direction preserving. Then, by using the definition of the Filippov set-valued map, one can show that $\nu_{i}=\mathbf{0}_{3}$ for all $\nu=\left[\nu_{1}, \ldots, \nu_{n}\right] \in \mathcal{F}_{2}(x)$ (recall that $x \in \mathcal{C}$ ). As $\mathcal{L}_{\mathcal{F}_{2}} V(x(t))$ is nonempty (by considering $t \in \Psi)$, there exists $a \in \mathcal{L}_{\mathcal{F}_{2}} V(x(t))$ such that $a=\zeta^{\top} \nu$ for all $\zeta \in \partial V(x(t))$, see the definition 77). By choosing $\zeta=e_{i} \otimes x_{i}(t)$, it follows that $a=\left(e_{i} \otimes\right.$ $\left.x_{i}\right)^{\top} \nu_{i}=0$, which implies that $\max \mathcal{L}_{\mathcal{F}_{2}} V(x(t)) \leqslant 0$. 
(ii) $|\mathcal{I}|=2$ and $\left|\mathcal{I}_{c}\right|=0$. In the following, we consider the Filippov solution of system (19), which can be written as

$$
\begin{aligned}
& \dot{x}_{1}=L_{x_{1}} \frac{x_{2}-x_{1}}{\left\|x_{2}-x_{1}\right\|}, \\
& \dot{x}_{2}=L_{x_{2}} \frac{x_{1}-x_{2}}{\left\|x_{1}-x_{2}\right\|} .
\end{aligned}
$$

Then it can be shown that, for $x_{1}=x_{2}$ (i.e., $x \in \mathcal{C}$ ), any element $\nu$ in the Filippov set-valued map of (25) satisfies $\nu_{1}=-\nu_{2}$. Stated differently, the following implication holds for $\nu=\left[\nu_{1}^{\top}, \nu_{2}^{\top}\right]^{\top}$ :

$$
\nu \in \mathcal{F}[h](x), x \in \mathcal{C} \Rightarrow \nu_{1}=-\nu_{2} .
$$

Next, by recalling that $\alpha(x)=\mathcal{I}$, it follows that

$$
\partial V(x)=\operatorname{co}\left\{e_{1} \otimes x_{1}, e_{2} \otimes x_{2}\right\}
$$

with $x_{1}=x_{2}$. Now, following a similar reasoning as in item $(i)$ on the basis of the definition of the set-valued Lie derivative in (7), it can be concluded that $a=\zeta^{\top} \nu=$ 0 , so that $\max \mathcal{L}_{\mathcal{F}_{1}} V(x(t))=0$ for all $x \in \mathcal{C}$.

Case 2: For $x \notin \mathcal{C}$, take an index $i \in \alpha(x)$ such that $\hat{L}_{i} x \neq \mathbf{0}_{3}$. Note that such $i$ indeed exists. Namely, assume in order to establish a contradiction that $\hat{L}_{i} x=\mathbf{0}_{3}$ for all $i \in \alpha(x)$. Then, it holds that

$$
0=x_{i}^{\top} \hat{L}_{i} x=\sum_{j \in N_{i}} x_{i}^{\top}\left(x_{i}-x_{j}\right) .
$$

Since $\left\|x_{i}\right\| \geqslant\left\|x_{j}\right\|$, it follows from (28) that $x_{j}=x_{i}$ for all $j \in N_{i}$. By repeating this argument and recalling that the interconnection topology is connected, it follows that $x_{j}=x_{i}$ for all $j \in \mathcal{I}$, i.e., $x \in \mathcal{C}$. This is a contradiction to $x \notin \mathcal{C}$.

For the index $i \in \alpha(x)$ satisfying $\hat{L}_{i} x \neq \mathbf{0}_{3}$, it follows from Assumption 1 that there exists $\gamma_{i}>0$ such that

$$
\mathcal{F}\left[f_{i}\right]\left(-\hat{L}_{i} x\right)=-\gamma_{i} \hat{L}_{i} x,
$$

i.e., for any $\nu \in \mathcal{F}_{2}(x)$ it holds that $\nu_{i}=-\gamma_{i} L_{x_{i}} \hat{L}_{i} x$. Note that this is a result of the direction-preserving property of either the vectorized signum function (for nonzero argument, then $\gamma_{i}=1$ ) or the Lipschitz continuous function (by Assumption 1). Then, choosing $\zeta \in \partial V(x)$ as $\zeta=e_{i} \otimes x_{i}$ (recall that $i \in \alpha(x)$ ), it follows that for any $\nu \in \mathcal{F}_{2}(x)$ we have

$$
\begin{aligned}
\zeta^{\top} \nu & =-\gamma_{i} x_{i}^{\top} L_{x_{i}} \hat{L}_{i} x \\
& =-\gamma_{i} x_{i}^{\top} \hat{L}_{i} x \\
& <0,
\end{aligned}
$$

where the second equality is based on $L_{x_{i}} x_{i}=x_{i}$.

Summarizing the results of the two cases leads to

$$
\max \mathcal{L}_{\mathcal{F}_{2}} V(x) \leqslant 0
$$

if $\left\|x_{i}\right\|<2 \pi$ for all $i \in \mathcal{I}$. Since the trajectory $x(t)$ is absolutely continuous, we have that if $\left\|x_{i}(0)\right\| \leqslant C<\pi$ for all $i \in \mathcal{I}$, all the trajectories remain within the set $\mathcal{S}_{1}(C)$.

Remark 1. As indicated in Example 1 sliding consensus can happen when $\mathcal{I}_{c}=\varnothing$ and $|\mathcal{I}|>2$. This will violate the strong invariance of the set $\mathcal{S}_{1}(C)$ with $C<\pi$, which will introduce singularity for the axis-angle representation for rotations.
Before we prove the finite-time convergence, we provide a sufficient condition for that all Filippov solutions of (19) converge to consensus asymptotically.

Lemma 3. Under the same assumptions of Lemma 2] all Filippov solutions of (19) asymptotically converge to static consensus.

Proof. Similar to the proof of Lemma 2, we shall prove that the conclusion holds for the bigger inclusion given by (24). In this proof cases $(i)$ and (ii) can be handled with the same arguments.

Consider the Lyapunov function candidate $V(x)=$ $\sqrt{x^{\top} \hat{L} x}$, which is convex, hence regular. The generalized gradient of $V$ is given as follows:

$$
\partial V(x)= \begin{cases}\frac{\hat{L} x}{\sqrt{x^{\top} \hat{L} x}} & \text { if } x \notin \mathcal{C}, \\ \operatorname{co}\left\{\lim _{y \rightarrow x} \frac{\hat{L} y}{\sqrt{y^{\top} \hat{L} y}}: y \notin \mathcal{C}\right\} & \text { if } x \in \mathcal{C} .\end{cases}
$$

Next we shall calculate the Lie derivative of $V$ by considering two cases.

(i) If $x \notin \mathcal{C}$, the Lie derivative is given as

$$
\begin{aligned}
\mathcal{L}_{\mathcal{F}_{2}} V(x) & =\frac{x^{\top} \hat{L}}{\sqrt{x^{\top} \hat{L} x}} \mathcal{F}_{2} \\
& =\frac{\sum_{i \in \mathcal{I}} x^{\top} \hat{L}_{i}^{\top} L_{x_{i}} \mathcal{F}\left[f_{i}\right]\left(-\hat{L}_{i} x\right)}{\sqrt{x^{\top} \hat{L} x}} .
\end{aligned}
$$

Here we have $\left(\hat{L}_{i} x\right)^{\top} L_{x_{i}} \mathcal{F}\left[f_{i}\right]\left(\hat{L}_{i} x\right) \geqslant 0$ for $i \in \mathcal{I}$. Indeed, it is because: (1), the conditions that the matrix $L_{x_{i}}^{1}>0$ for $x_{i}$ satisfying $\left\|x_{i}\right\|<\pi$; (2), Assumption 1 about direction preservation, and (3), the set $\mathcal{S}_{1}(C)$ is strongly invariant for $C<\pi$. Moreover, if $\hat{L}_{i} x \neq \mathbf{0}_{3}$, the set $\left(\hat{L}_{i} x\right)^{\top} L_{x_{i}} \mathcal{F}\left[f_{i}\right]\left(\hat{L}_{i} x\right) \subset \mathbb{R}_{>0}$. Hence $\mathcal{L}_{\mathcal{F}_{2}} V \subset \mathbb{R}_{<0}$.

(ii) If $x \in \mathcal{C}$, it can be seen that $\zeta \in \partial V(x)$ implies $-\zeta \in$ $\partial V(x)$. Hence if $\mathcal{L}_{\mathcal{F}_{2}} V \neq \varnothing$, it has to be $\{0\}$. In fact, by taking $\mathbf{0} \in \mathcal{F}_{2}$, we have that $0 \in \mathcal{L}_{\mathcal{F}_{2}} V$.

Next, by Theorem 3 in [9], it holds that all Filippov solutions of (24) converge to the set $\overline{Z_{\mathcal{F}_{2}, V}}$ asymptotically. The remaining task is to characterize the set $Z_{\mathcal{F}_{2}, V}$. So far we have shown that $x \notin Z_{\mathcal{F}_{2}, V} \forall x \notin \mathcal{C}$, which implies that $Z_{\mathcal{F}_{2}, V} \subset \mathcal{C}$. By the fact that $\mathcal{C}$ is closed, we have $\overline{Z_{\mathcal{F}_{2}, V}} \subset \mathcal{C}$. Moreover, when $x \in \mathcal{C}, \dot{x}_{i}=0$ where $i \in \mathcal{I}_{c}$, which implies that $x_{i}$ remains constant. In conclusion, asymptotic convergence to static consensus is guaranteed.

Now we are ready for the main result of this section.

Theorem 4. Assume that $R_{i}(0) \in B_{\pi}(I) \subset S O(3)$ for all $i \in \mathcal{I}$ and that the graph $\mathcal{G}$ is connected. Consider the multi-agent system (18) satisfying Assumption 1 and the corresponding differential inclusion (19). Then, all Filippov solutions converge to consensus in finite time if one of the following conditions holds:

(i) $|\mathcal{I}|>2$ and $\left|\mathcal{I}_{c}\right|=1$;

(ii) $|\mathcal{I}|=2$ and $\left|\mathcal{I}_{c}\right| \leqslant 1$.

Proof. The proof is separated into two parts, one for each of the conditions. 
(i) Without loss of generality, we assume that $f_{1}$ satisfies the condition $(i)$ in Assumption 1 while $f_{2}, \ldots, f_{n}$ are sign. Similar to Lemma 2, instead of proving the conclusion for the differential inclusion (19), we shall show that it holds for the bigger inclusion given by (24).

Consider the Lyapunov function candidate $V(x)=$ $\sqrt{x^{\top} \hat{L} x}$. We shall show that there exists $c$ such that $\max \mathcal{L}_{\mathcal{F}_{2}} V<c<0$ for any initial condition $\mathcal{S}_{1}(C) \backslash \mathcal{C}$ with $C<\pi$.

In the proof of Lemma 3, we have shown that for $x \notin \mathcal{C}$, the Lie derivative is given by (33). By the fact that $L_{x_{i}}^{1}>0$ with $\left\|x_{i}\right\|<\pi$ and $f_{i}$ is direction preserving, we have

$$
\mathcal{L}_{\mathcal{F}_{2}} V \leqslant \frac{\sum_{i=2}^{n} x^{\top} \hat{L}_{i}^{\top} L_{x_{i}} \mathcal{F}[\operatorname{sign}]\left(-\hat{L}_{i} x\right)}{\sqrt{x^{\top} \hat{L} x}} .
$$

Furthermore, the Filippov set-valued map

$$
\mathcal{F}[\operatorname{sign}]\left(-\hat{L}_{i} x\right)= \begin{cases}\left\{\frac{-\hat{L}_{i} x}{\left\|\hat{L}_{i} x\right\|}\right\} & \text { if }\left\|\hat{L}_{i} x\right\| \neq 0, \\ \{v \mid\|v\| \leqslant 1\} & \text { if }\left\|\hat{L}_{i} x\right\|=0,\end{cases}
$$

which implies that

$$
\begin{aligned}
& x^{\top} \hat{L}_{i}^{\top} L_{x_{i}} \mathcal{F}[\operatorname{sign}]\left(-\hat{L}_{i} x\right) \\
= & \begin{cases}\left\{\frac{-x^{\top} \hat{L}_{i}^{\top} L_{x_{i}} \hat{L}_{i} x}{\left\|\hat{L}_{i} x\right\|}\right\} & \text { if }\left\|\hat{L}_{i} x\right\| \neq 0, \\
\left\{\left\|\hat{L}_{i} x\right\|\right\} & \text { if }\left\|\hat{L}_{i} x\right\|=0 .\end{cases}
\end{aligned}
$$

Note that, for any $x$ satisfying $\left\|x_{i}\right\|<\pi$ for all $i \in \mathcal{I}$, there exists $c_{1} \in(0,1)$, which only depends on $\max _{i}\left\|x_{i}(0)\right\|$, such that $L_{x_{i}}-c_{1} I \geqslant 0$ for all $i \in \mathcal{I}$. This implies that

$$
x^{\top} \hat{L}_{i}^{\top} L_{x_{i}} \mathcal{F}[\operatorname{sign}]\left(-\hat{L}_{i} x\right) \subset\left(-\infty,-c_{1}\left\|\hat{L}_{i} x\right\|\right] .
$$

So far we have shown that, for any $a \in \mathcal{L}_{\mathcal{F}_{2}} V(x)$, it holds that

$$
a \leqslant-c_{1} \frac{\sum_{i=2}^{n}\left\|\hat{L}_{i} x\right\|}{\sqrt{x^{\top} \hat{L} x}} .
$$

Furthermore, by using that $\hat{L}_{1} x=-\sum_{i=2}^{n} \hat{L}_{i} x$, which is based on the connectivity of the graph $\mathcal{G}$, we have

$$
\left\|\hat{L}_{1} x\right\|=\left\|\sum_{i=2}^{n} \hat{L}_{i} x\right\| \leqslant \sum_{i=2}^{n}\left\|\hat{L}_{i} x\right\|,
$$

where the triangle inequality is used. Then, the use of (39) in (38) yields

$$
a \leqslant-\frac{c_{1}}{2} \frac{1}{\sqrt{x^{\top} \hat{L} x}}\left(\sum_{i=1}^{n}\left\|L_{i} x\right\|\right) .
$$

By exploiting the observation that $L$ is a graph Laplacian, it holds that

$$
L=U^{\top} \Lambda U, \quad L^{\top} L=U^{\top} \Lambda^{2} U,
$$

where $\Lambda=\operatorname{diag}\left\{0, \lambda_{2}, \ldots, \lambda_{n}\right\}$ is a diagonal matrix with real-valued eigenvalues satisfying $0<\lambda_{2}$ and $\lambda_{j} \leqslant \lambda_{j+1}$ for $j=2, \ldots, n$. The matrix $U$ collects the corresponding right-eigenvectors. From (41), it can be seen that

$$
L^{\top} L-c L \geqslant 0
$$

for any $c \in\left[0, \lambda_{2}\right]$. Consequently, using $\hat{L}=L \otimes I$, it follows that

$$
\left(\sum_{i=1}^{n}\left\|\hat{L}_{i} x\right\|\right)^{2}=x^{\top} \hat{L}^{\top} \hat{L} x \geqslant c x^{\top} \hat{L} x .
$$

After taking the square root (note that $x^{\top} L x>0$ for all $x \notin \mathcal{C}$ ) in (43) and substituting the result in (40), the result

$$
a \leqslant-\frac{c_{1} \sqrt{c}}{2} \frac{\sqrt{x^{\top} \hat{L} x}}{\sqrt{x^{\top} \hat{L} x}}=-\frac{c_{1} \sqrt{c}}{2}
$$

follows, which proves finite-time convergence to consensus by Proposition 4 in [9] and Lemma 3.

(ii) By using a similar reasoning, we have that for any $a \in \mathcal{L}_{\mathcal{F}[\hat{h}]} V(x)$, it satisfies that $a \leqslant-c_{1}$ where $c_{1}$ satisfying $L_{x_{i}}-c_{1} I \geqslant 0$ for all $i \in \mathcal{I}$. This again implies finite time convergence.

Remark 2. Theorem 4 provides sufficient conditions for finitetime convergence of the protocol (17) satisfying Assumption 1. However, we conjecture these sufficient conditions to be necessary as well. Namely, for the case $|\mathcal{I}|>2$, we expect that all the Filippov solutions of (19) converge to consensus in finite time if and only if $\left|\mathcal{I}_{c}\right|=1$; and for the case $|\mathcal{I}|=2$, we expect that the finite-time synchronization is achieved if and only if $\left|\mathcal{I}_{c}\right| \leqslant 1$. We show that the latter statement holds according to the following argument.

If $|\mathcal{I}|=2$ and $\left|\mathcal{I}_{c}\right|>1$, then $\mathcal{I}=\mathcal{I}_{c}$. In this case, we can only have asymptotic convergence if $\mathcal{I}=\mathcal{I}_{c}$. Indeed, the right-hand side of (18) is Lipschitz; therefore, finite-time convergence to an equilibrium can not occur.

Unfortunately, for the case $|\mathcal{I}|>2$, we can not prove the necessity, which leaves it as an open question.

We close this subsection by demonstrating the result in Theorem 4 and conjecture in Remark 2 by an example.

Example 2. Consider the three-agent system

$$
\begin{aligned}
& \dot{x}_{1}=L_{x_{1}}\left(x_{2}-x_{1}\right) \\
& \dot{x}_{2}=L_{x_{2}} \operatorname{sign}\left(x_{1}+x_{3}-2 x_{2}\right) \\
& \dot{x}_{3}=L_{x_{3}} \operatorname{sign}\left(x_{2}-x_{3}\right),
\end{aligned}
$$

defined on a line graph with. Notice that this system meets condition (i) in Theorem 4. A phase portrait and trajectory of this system are depicted in Fig. 1. There, we can see that finite-time consensus is achieved.

Next, modify the system to

$$
\begin{aligned}
& \dot{x}_{1}=L_{x_{1}}\left(x_{2}-x_{1}\right) \\
& \dot{x}_{2}=L_{x_{2}} \operatorname{sign}\left(x_{1}+x_{3}-2 x_{2}\right) \\
& \dot{x}_{3}=L_{x_{3}}\left(x_{2}-x_{3}\right) .
\end{aligned}
$$

Notice that $\mathcal{I}_{c}=\{1,3\}$, hence the conditions (i) and (ii) in Theorem 4 are not satisfied. As stated in Remark 2 we expect there are some trajectories that only converge to consensus asymptotically, but not in finite time. In fact, we construct such a solution as follows. For the initial condition satisfying $x_{1}(0)+x_{3}(0)=x_{2}(0)=\mathbf{0}$, the trajectory 


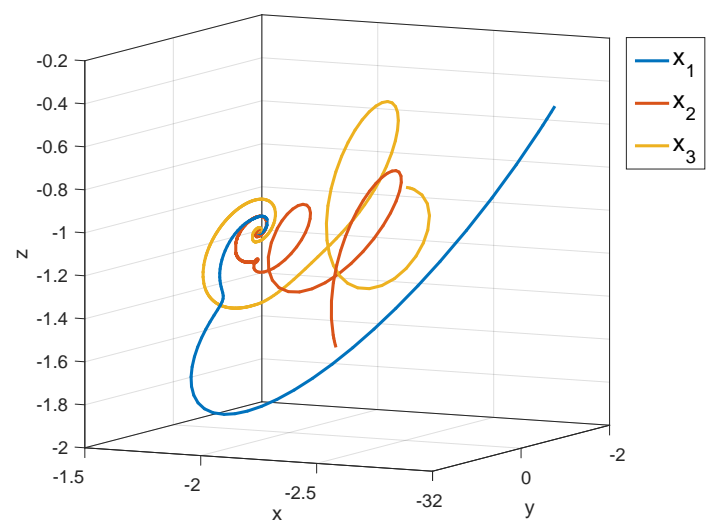

(a) Phase portraits of $x_{i}, i=1,2,3$ in $\mathbb{R}^{3}$.

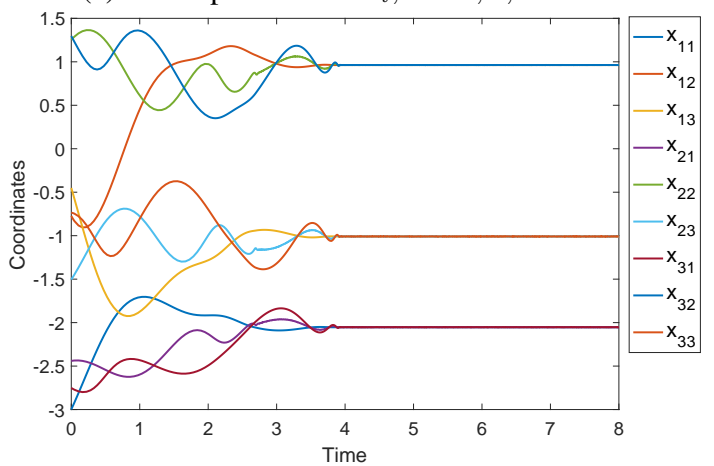

(b) Evolution of the three coordinates of $x_{i}, i=1,2,3$. Finite-time consensus is achieved.

Fig. 1: The simulation of Example 2.

$x_{1}(t)=x_{1}(0) e^{-t}, x_{2}(t)=\mathbf{0}, x_{3}(t)=x_{3}(0) e^{-t}$ is a Filippov solution of 46. Indeed, the trajectory obeys the dynamic

$$
\left(\begin{array}{c}
\dot{x}_{1} \\
\dot{x}_{2} \\
\dot{x}_{3}
\end{array}\right)=\left(\begin{array}{c}
-x_{1} \\
\mathbf{0} \\
-x_{3}
\end{array}\right) \in \mathcal{F}\left[\left(\begin{array}{c}
L_{x_{1}}\left(x_{2}-x_{1}\right) \\
L_{x_{2}} \operatorname{sign}\left(x_{1}+x_{3}-2 x_{2}\right) \\
L_{x_{3}}\left(x_{2}-x_{3}\right)
\end{array}\right)\right](x)
$$

where we used that $L_{x_{2}}=I, L_{x_{i}} x_{i}=x_{i}$, and $\mathbf{0} \in \mathcal{F}[\operatorname{sign}](\mathbf{0})$. Hence we have a trajectory converging to consensus only asymptotically.

\section{B. Control law for local convergence}

In this subsection, we consider another type of controller to achieve finite-time synchronization. The controller has a finite number of control actions. However, differently than controller (17), the controller in this subsection only guarantees local convergence.

We consider the discontinuous control protocol

$$
\omega_{i}=\sum_{j \in N_{i}} \operatorname{sign}_{c}\left(x_{j}-x_{i}\right)
$$

where $\operatorname{sign}_{c}$ is defined in 9). Notice that each $\omega_{i}$ only takes a finite number of values. Now the closed-loop dynamic is

$$
\dot{x}_{i}=L_{x_{i}} \sum_{j \in N_{i}} \operatorname{sign}_{c}\left(x_{j}-x_{i}\right) .
$$

The compact version of the system (48) can be written as

$$
\dot{x}=-L_{x} \hat{B} \operatorname{sign}_{c}\left(\hat{B}^{\top} x\right),
$$

where $B$ is the incidence matrix of the underlying graph and $\hat{B}=B \otimes I_{3}$. Similar to the previous subsection, we understand the solution of 49 in the sense of Filippov, namely solutions of the following differential inclusion:

$$
\begin{aligned}
\dot{x} & \in \mathcal{F}\left[-L_{x} \hat{B} \operatorname{sign}_{c}\left(\hat{B}^{\top} x\right)\right](x) \\
& =-L_{x} \hat{B} \mathcal{F}\left[\operatorname{sign}_{c}\left(\hat{B}^{\top} x\right)\right](x) \\
& =: \mathcal{F}_{3}(x),
\end{aligned}
$$

where the first equality is based on Theorem 1(5) in [22] and the fact that $L_{x_{i}}$ is continuous for $\left\|x_{i}\right\| \in[0, \pi)$. By using Theorem 1 [22], we can enlarge the differential inclusion $\mathcal{F}_{3}$ as follows:

$$
\begin{aligned}
\mathcal{F}_{3}(x) & \subset-L_{x} \hat{B} \underset{i=1}{\times 3 n} \mathcal{F}\left[\operatorname{sign}_{c}\right]\left(\left(\hat{B}^{\top}\right)_{i} x\right) \\
& =: \mathcal{F}_{4}(x),
\end{aligned}
$$

where $\left(\hat{B}^{\top}\right)_{i}$ is the $i$ th row of $\hat{B}^{\top}$ and the set-valued function $\mathcal{F}\left[\operatorname{sign}_{c}\right]$ is defined as

$$
\mathcal{F}\left[\operatorname{sign}_{c}\right](x)= \begin{cases}1 & \text { if } x>0 \\ {[-1,1]} & \text { if } x=0 \\ -1 & \text { if } x<0\end{cases}
$$

Before we show the main result, we present a compact strongly invariant set.

Lemma 5. The set $\mathcal{S}_{2}(C)=\left\{x \in \mathbb{R}^{3 n} \mid \sum_{i=1}^{n}\left\|x_{i}\right\|_{2}^{2}<C\right\}$ with $C<4 \pi^{2}$ is strongly invariant for the differential inclusion (50). Moreover, all the solutions of system (50) converge to consensus asymptotically.

Proof. Consider the Lyapunov function candidate $V(x)=$ $\frac{1}{2} x^{\top} x=\frac{1}{2} \sum_{i=1}^{n} x_{i}^{\top} x_{i}$. We shall show that the conclusion holds for the bigger inclusion $\mathcal{F}_{4}$ defined in (51).

Since $V$ is smooth, the set-valued Lie-derivative $\mathcal{L}_{\mathcal{F}_{4}} V(x)$ is given as

$$
\begin{aligned}
\mathcal{L}_{\mathcal{F}_{4}} V(x) & =x^{\top} \mathcal{F}_{4}(x) \\
& =-x^{\top} \hat{B} \underset{i=1}{\times} \underset{\mathcal{X}}{\times}\left[\operatorname{sign}_{c}\right]\left(\left(\hat{B}^{\top}\right)_{i} x\right),
\end{aligned}
$$

where the last equality is implied by that $L_{x_{i}}$ is well-defined when $\left\|x_{i}\right\|<2 \pi$, which is satisfied by the elements in $\mathcal{S}_{2}(C)$, and $x_{i}^{\top} L_{x_{i}}=x_{i}^{\top}$. Furthermore, note that

$$
\begin{aligned}
& -x^{\top} \hat{B} \underset{i=1}{\times 3 n} \mathcal{F}\left[\operatorname{sign}_{c}\right]\left(\left(\hat{B}^{\top}\right)_{i} x\right) \\
& =-\sum_{(i, j) \in \mathcal{E}}\left(x_{i}-x_{j}\right)^{T} \underset{k=1}{\stackrel{3}{\times}} \mathcal{F}\left[\operatorname{sign}_{c}\right]\left(x_{i_{k}}-x_{j_{k}}\right) \\
& \subset \mathbb{R}_{\leqslant 0},
\end{aligned}
$$

which indicates that $V(x(t))$ is not increasing along the trajectories when $C<4 \pi^{2}$. Hence the set $\mathcal{S}_{2}(C)$ is strongly invariant. Notice that the boundedness of the trajectories is also guaranteed.

Finally, by Theorem 3 in [9], we have that the Filippov solution of system (50) will asymptotically converge to the set

$$
\Omega=\overline{\left\{x \in \mathbb{R}^{3 n} \mid 0 \in \mathcal{L}_{\mathcal{F}_{4}} V(x)\right\}} .
$$


By (54) it is straightforward to verify that $\Omega=\mathcal{C}$. Then the conclusion follows.

From the previous lemma, we note that the continuity assumption, i.e., Assumption 1 ( $i$ ), is not needed for controller 47). However, the control law 47) can only guarantee local convergence as indicated in the following Theorem and the complete proof can be found in [33].

Theorem 6. Assume that the initial rotations of the agents satisfy $\sum_{i=1}^{n} d_{R}^{2}\left(I, R_{i}(0)\right)<\pi^{2}$ and the underlying graph $\mathcal{G}$ is connected. Consider the multi-agent system (49) and the corresponding differential inclusion (50). Then, attitude synchronization is achieved in finite time.

\section{CONCLUSiON}

In this paper, we considered the finite-time attitude synchronization problem of multi-agent systems. Two finite-time consensus control protocols were proposed. The first protocol guaranteed global convergence in the sense that the initial rotation of each agent can be arbitrary in an open ball of radius $\pi$, which contains all but a set of measure zero of the rotations in $S O(3)$. In addition, we proposed a second protocol based on binary control, which achieves local convergence to the consensus subspace, in the sense that the initial rotations have to be close enough to the origin in $S O(3)$. For these two controllers, sufficient conditions were presented to guarantee finite-time convergence and boundedness of the solutions. Future studies include further investigation on the necessity of these conditions. Furthermore, the results in this paper based on absolute rotation measurements of the agents, hence finitetime synchronization protocols using relative measurements is another future topic.

\section{REFERENCES}

[1] A. Bacciotti and F. Ceragioli. Stability and stabilization of discontinuous systems and nonsmooth Lyapunov functions. ESAIM: Control, Optimisation and Calculus of Variations, 4:361-376, 1999.

[2] N. Athanasopoulos, M. Lazar, C. Böhm, and F. Allgöwer. On stability and stabilization of periodic discrete-time systems with an application to satellite attitude control. Automatica, 50(12):3190-3196, 2014.

[3] S. Bhat and D. Bernstein. A topological obstruction to continuous global stabilization of rotational motion and the unwinding phenomenon. Systems \& Control Letters, 39(1):63-70, 2000.

[4] B. Bollobas. Modern Graph Theory, volume 184 of Graduate Texts in Mathematics. Springer, New York, 1998.

[5] J. Bower and G. Podraza. Digital implementation of time-optimal attitude control. IEEE Transactions on Automatic Control, 9(4):590$591,1964$.

[6] R. W. Brockett. Asymptotic stability and feedback stabilization. In Differential Geometric Control Theory (R. W. Brockett, R. S. Millman and H. J. Sussmann, Eds), pages 181-191. Birkhauser, Boston, 1983.

[7] G. Chen, F. L. Lewis, and L. Xie. Finite-time distributed consensus via binary control protocols. Automatica, 47(9):1962 - 1968, 2011.

[8] F. H. Clarke. Optimization and Nonsmooth Analysis. Classics in Applied Mathematics. Society for Industrial and Applied Mathematics, 1990.

[9] J. Cortés. Finite-time convergent gradient flows with applications to network consensus. Automatica, 42(11):1993-2000, 2006.

[10] J. Cortés. Discontinuous dynamical systems. Control Systems, IEEE, 28(3):36-73, 2008

[11] Y. Dong and Y. Ohta. Attitude synchronization of rigid bodies via distributed control. In Proceedings of the 55th IEEE Conference on Decision and Control, pages 3499-3504. IEEE, 2016.

[12] H. Du, S. Li, and C. Qian. Finite-time attitude tracking control of spacecraft with application to attitude synchronization. IEEE Transactions on Automatic Control, 56(11):2711-2717, Nov 2011.
[13] A.F. Filippov and F.M. Arscott. Differential Equations with Discontinuous Righthand Sides: Control Systems. Mathematics and its Applications. Springer, 1988.

[14] Q. Hui, W. M. Haddad, and S. P. Bhat. Finite-time semistability, Filippov systems, and consensus protocols for nonlinear dynamical networks with switching topologies. Nonlinear Analysis: Hybrid Systems, 4(3):557_ $573,2010$.

[15] H. Kowalik. A spin and attitude control system for the Isis-I and Isis-B satellites. Automatica, 6(5):673-682, sep 1970.

[16] T. Lee. Relative attitude control of two spacecraft on $\operatorname{SO}(3)$ using line-of-sight observations. In Proceedings of IEEE American Control Conference, pages 167-172, 2012.

[17] T. Lee. Global exponential attitude tracking controls on $\mathrm{SO}(3)$. IEEE Transactions on Automatic Control, 60(10):2837-2842, 2015.

[18] J. Li and K. D. Kumar. Decentralized fault-tolerant control for satellite attitude synchronization. IEEE Transactions on Fuzzy Systems, 20(3):572-586, 2012.

[19] X. Liu, J. Lam, W. Yu, and G. Chen. Finite-time consensus of multiagent systems with a switching protocol. IEEE Transactions on Neural Networks and Learning Systems, 27(4):853-862, 2016.

[20] Y. Ma, S. Soatto, J. Kosecka, and S. Sastry. An Invitation to 3-D Vision: From Images To Geometric Models, volume 26. Springer Science \& Business Media, 2012.

[21] R. Murray, Z. Li, and S. Sastry. A Mathematical Introduction To Robotic Manipulation. CRC press, 1994

[22] B. Paden and S. Sastry. A calculus for computing Filippov's differential inclusion with application to the variable structure control of robot manipulators. IEEE Transactions on Circuits and Systems, 34(1):7382, 1987.

[23] P.O. Pereira, D. Boskos, and D.V. Dimarogonas. A common framework for attitude synchronization of unit vectors in networks with switching topology. In Proceedings of the 55th IEEE Conference on Decision and Control, pages 3530-3536, 2016.

[24] K.Y. Pettersen and O. Egeland. Position and attitude control of an underactuated autonomous underwater vehicle. In Proceedings of the 35th IEEE Conference on Decision and Control, volume 1, pages $987-$ 991. IEEE, 1996.

[25] W. Ren. Distributed cooperative attitude synchronization and tracking for multiple rigid bodies. IEEE Transactions on Control Systems Technology, 18(2):383-392, 2010.

[26] A. Sarlette, R. Sepulchre, and N. E. Leonard. Autonomous rigid body attitude synchronization. Automatica, 45(2):572-577, 2009.

[27] H. Schaub and J. L. Junkins. Analytical Mechanics of Space Systems. AIAA Education Series, Reston, VA, 2003.

[28] W. Song, J. Markdahl, X. Hu, and Y. Hong. Distributed control for intrinsic reduced attitude formation with ring inter-agent graph. In Proceedings of the 54th IEEE Conference on Decision and Control, pages 5599-5604, 2015.

[29] J. Thunberg, J. Goncalves, and X. Hu. Consensus and formation control on se (3) for switching topologies. Automatica, 66:109-121, 2016.

[30] J. Thunberg, W. Song, E. Montijano, Y. Hong, and X. Hu. Distributed attitude synchronization control of multi-agent systems with switching topologies. Automatica, 50(3):832 - 840, 2014.

[31] R. Tron, B. Afsari, and R. Vidal. Intrinsic consensus on $\mathrm{SO}(3)$ with almost-global convergence. In Proceedings of the 51st IEEE Conference on Decision and Control, pages 2052-2058, 2012.

[32] P. Tsiotras and J. M. Longuski. Spin-axis stabilization of symmetric spacecraft with two control torques. Systems \& Control Letters, 23(6):395-402, 1994.

[33] J. Wei, S. Zhang, A. Adaldo, X. Hu, and K. H. Johansson. Finite-time attitude synchronization with a discontinuous protocol. In Proceedings of the 13th IEEE International Conference on Control and Automation, 2017.

[34] T. Wu, B. Flewelling, F. Leve, and T. Lee. Spacecraft relative attitude formation tracking on so (3) based on line-of-sight measurements. In Proceedings of IEEE American Control Conference, pages 4820-4825, 2013.

[35] Q. Zong and S. Shao. Decentralized finite-time attitude synchronization for multiple rigid spacecraft via a novel disturbance observer. ISA Transactions, 65:150 - 163, 2016. 